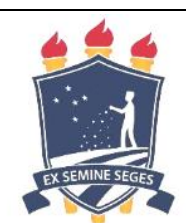

\title{
Avaliação do potencial de cicatrização do óleo de semente de girassol associado ao ultrassom terapêutico em ratos (Rattus novergicus) com ferida cutânea induzida
}

\author{
[Evaluation of the healing potential of sunflower seed oil associated with therapeutic ultrasound in \\ rats (Rattus novergicus) with induced skin wound]
}

\section{"Artigo Científico/Scientific Article"}

\author{
Luiggi Adriani de Lima e Montier Liciarde ${ }^{1}$, Célia Araújo da Silva² ${ }^{2}$ Fábia Regina Nascimento \\ Fernando Burgos $^{3 *}$, Edvaldo Lopes de Almeida ${ }^{4}$
}

\author{
${ }^{1}$ Médico-veterinário autônomo. Recife-PE, Brasil. \\ ${ }^{2}$ Fisioterapeuta autônoma. Recife-PE, Brasil. \\ ${ }^{3}$ Departamento de Morfologia e Fisiologia Animal, Universidade Federal Rural de Pernambuco, Recife-PE, Brasil. \\ ${ }^{4}$ Departamento de Medicina Veterinária, Universidade Federal Rural de Pernambuco, Recife-PE, Brasil. \\ *Autor para correspondência/Corresponding author: E-mail: fabia.burgos@ufrpe.br
}

\section{Resumo}

Com este experimento objetivou-se avaliar o potencial de cicatrização do óleo de semente de girassol associado ao ultrassom terapêutico em ratos (Rattus novergicus) com ferida cutânea induzida. Utilizou-se 24 ratos adultos da linhagem Wistar, machos, distribuídos aleatoriamente em quatro grupos de seis animais, onde no grupo I (controle) se realizou a aplicação de água destilada; no grupo II foi aplicado óleo de semente de girassol; no grupo III foi realizada uma intervenção com Ultrassom $3 \mathrm{MHz}$ após a aplicação de óleo de semente de girassol e no grupo IV apenas a aplicação de ultrassom. De acordo com o teste F, foram encontradas evidências de diferenças significativas, ao nível de $1 \%$ de probabilidade, entre os tratamentos, com relação ao tempo médio de cicatrização, onde houve grande significância da diferença nos tempos de cicatrização do Grupo III em relação aos outros grupos. Concluiu-se, com a presente pesquisa que o ultrassom terapêutico favoreceu a permeabilidade do óleo de semente de girassol nas estruturas teciduais, contribuindo para o menor tempo de cicatrização em feridas cutâneas experimentais de ratos (Rattus novergicus).

Palavras-chave: ultrassom; óleo de girassol; pele.

\begin{abstract}
This experiment aimed to evaluate the healing potential of sunflower seed oil associated with therapeutic ultrasound in rats (Rattus novergicus) with induced skin wound. Twenty-four adult male Wistar rats were used, randomly distributed in four groups of six animals, where in group I (control), distilled water was applied; in group II, sunflower seed oil was applied; in group III, an intervention with Ultrasound $3 \mathrm{MHz}$ was performed after the application of sunflower seed oil; and in group IV, only ultrasound was performed. According to the F test, evidence of significant differences was found, at the level of $1 \%$ probability, between treatments, with respect to the average healing time, where there was high significance in the difference in the healing times of Group III in relation to the other groups. It was concluded, with the present research, that the therapeutic ultrasound favored the permeability of sunflower seed oil in the tissue structures, contributing to the shorter healing time in experimental skin wounds of rats (Rattus novergicus).
\end{abstract}

Keywords: ultrasound; sunflower seed oil; skin.

\section{Introdução}

Segundo Roger et al. (2019) a pele é o maior

órgão do corpo humano e representa aproximadamente $16 \%$ do peso corporal total.
Compreende a interface primária entre os ambientes interno e externo, atuando como barreira de proteção a uma série de estressores ambientais, assim como mantém a homeostase, para evitar a 
perda de água e eletrólitos (Roger et al., 2019). Uma ferida consiste em qualquer interrupção na continuidade de um tecido corpóreo, em maior ou menor extensão, causada principalmente por trauma ou desencadeada por uma afecção clínica (Leite et al., 2012).

O ultrassom tem sido amplamente utilizado como transportador de fármacos aplicados topicamente, pois seus mecanismos físicos alteram a permeabilidade tissular, favorecendo o processo difusional destes medicamentos para o subcutâneo (Kitchen e Partridge, 1990). O ultrassom terapêutico (UST) é uma técnica frequentemente utilizada na fisioterapia para tratamento de lesões em tecidos moles, inflamações, distúrbios circulatórios e estimulação da reparação tecidual (Benson e McElnay, 1988). Na terapia ultrassônica a frequência utilizada é de 0,7 a $3 \mathrm{Mhz}$ (Marks et al., 2000).

Matheus et al. (2008) concluíram que a intervenção terapêutica por meio de ultrassom aumentou as propriedades mecânicas dos músculos lesionados, onde não foram observadas diferenças significativas entre o grupo tratado com frequência de $1 \mathrm{MHz}$ e aquele que recebeu uma frequência de 3 MHz. Apesar dos resultados positivos encontrados neste estudo, é fundamental continuar a pesquisa em ultrassom terapêutico, com o objetivo de criar protocolos, bem como determinar parâmetros mais precisos para cada indivíduo, que reforçará a validade científica deste procedimento.

Em especial, o UST é produzido por uma corrente alternada que flui por um cristal piezoelétrico, alojado em um transdutor que gera uma energia sonora. Esta, quando aplicada nos tecidos biológicos, é capaz de produzir alterações celulares por efeitos mecânicos. É um recurso amplamente utilizado na prática clínica, demonstrando resultados positivos no reparo tecidual, entretanto, os seus parâmetros ainda vêm sendo investigados (Mendonça et al., 2006).

Dentro deste contexto terapêutico, podemos observar a abrangência das ondas ultrassônicas a uma variedade de condições, com alegações de resultados bem-sucedidos. Quando as ondas acústicas penetram no corpo, podem exercer um efeito sobre as células e tecidos mediante mecanismos físicos térmicos e atérmicos (Young, 1990).

Korelo e Fernandes (2016) realizaram uma pesquisa bibliográfica e concluíram que apesar de divergências entre estudos, existe evidência limitada da HFU (High Frequency Ultrasound), e evidência moderada da NCLFU (Noncontact Low Frequency Ultrasound) como recurso adjuvante para o tratamento de feridas; mesmo que a base científica não consiga responder com clareza a relação entre dose-resposta. $\mathrm{O}$ resultado desta pesquisa amplia a base de conhecimentos sobre a utilização do ultrassom terapêutico e suporta a ideia de que, entre as formas utilizadas, NCLFU produz resultados benéficos para o tratamento de feridas.

O uso de plantas como medicamento terapêutico é tão antigo quanto a espécie humana, que busca com preferência remédios naturais e eficazes, proporcionando uma melhoria na qualidade de vida e oferecendo outra forma de tratamento além dos medicamentos alopáticos. Óleos de origem vegetal são extraídos de diversas partes das plantas, na alimentação eles podem fornecer vitaminas e ácidos graxos essenciais, que possuem propriedades bactericidas, antissépticas e anti-inflamatórias, por meio de aplicações cosméticas e fitoterápicas tratam alergias e feridas. A associação sinérgica dos ácidos linoleicos, linolênico e oleico, fazem do óleo de girassol um produto potencial a ser utilizado na terapia de feridas (Sousa, 2018).

Marques et al. (2004) demonstraram os efeitos do uso de óleo de semente de girassol no tratamento de feridas cutâneas de dezoito carneiros da raça Santa Inês, que foram divididos em três grupos de acordo com o pós-cirúrgico. $\mathrm{O}$ uso tópico do óleo de semente de girassol incrementou o tempo de cicatrização, podendo ser utilizado como alternativa terapêutica em feridas por segunda intenção.

O tratamento tópico com óleos de café levou a ações locais e sistêmicas e à cicatrização mais rápida de feridas em ratos. O tratamento com óleo de girassol induziu efeitos tópicos e sistêmicos. Como o óleo é amplamente utilizado para diminuir o tempo de cicatrização de úlceras, novos estudos são necessários para avaliar a segurança do tratamento e possíveis efeitos indesejados do uso tópico de óleo vegetal para lesões cutâneas (Lania, 2018).

Objetivou-se analisar a retração da ferida cutânea de ratos avaliando os efeitos do uso do ultrassom associado ao óleo de semente de girassol, aplicados isolados e associados.

\section{Material e Métodos}

Utilizou-se um total de 24 ratos (Rattus novergicus var. albinus, Rodentia, Mammalia), 
clinicamente sadios, adultos, da linhagem Wistar, machos, com massa corporal de $308 \pm 32 \mathrm{~g}$ provenientes do Biotério do Departamento de Morfologia e Fisiologia Animal da Universidade Federal Rural de Pernambuco.

Os animais foram distribuídos aleatoriamente em quatro grupos com seis ratos e submetidos ao tratamento diário por 30 dias. Onde no grupo I (controle) se realizou a aplicação com auxílio de seringa de $1 \mathrm{ml}$ de água destilada; no grupo II foi aplicado com auxílio de seringa $1 \mathrm{ml}$ de óleo de semente de girassol (Tabela 1); no grupo III foi realizada uma intervenção com Ultrassom 3 MHz após a aplicação de $1 \mathrm{ml}$ óleo de semente de girassol com auxílio de seringa; e no grupo IV apenas a intervenção com ultrassom (Tabela 2).

Tabela 1. Características do óleo de semente de girassol.

\begin{tabular}{ll}
\hline SKU & 990 \\
\hline Para uso & Alimentício, cosmético \\
Parte utilizado & Semente \\
Tipo de Óleo & Vegetal \\
Características do Óleo & $100 \%$ puro e natural, extra virgem, filtrado, prensado a frio \\
CAS/EINECS & $8001-21-6$ \\
Marca & Embrafarma / Pharma Face \\
\hline
\end{tabular}

Tabela 2. Descrição dos grupos com seus respectivos procedimentos.

\begin{tabular}{ll}
\hline Grupo & Procedimento a cada 24 horas \\
\hline I (controle) & $1,0 \mathrm{~mL}$ de água destilada sobre a ferida \\
II & $1,0 \mathrm{~mL}$ de óleo de semente de girassol sobre a ferida \\
III & ondas ultrassônicas $(3 \mathrm{MHz})$ sobre a ferida protegida por papel filme e em seguida instilado \\
& $1,0 \mathrm{~mL}$ de óleo de girassol \\
IV & ondas ultrassônicas $(3 \mathrm{MHz})$ sobre a ferida protegida por papel filme \\
\hline
\end{tabular}

Os animais foram acondicionados individualmente em gaiolas de policarbonato forradas com maravalha de pinus e tampa gradeada de aço. As gaiolas tiveram sua tampa colocada de maneira invertida, presas com arame de aço inoxidável, para evitar lesões no animal. Cada gaiola identificada com ficha e prontuário. Realizou-se controle de ciclo claro/escuro (12 horas/12 horas) e forneceu-se acesso à água e ração ad libitum.

Para a realização da ferida experimental, os animais foram submetidos à anestesia dissociativa

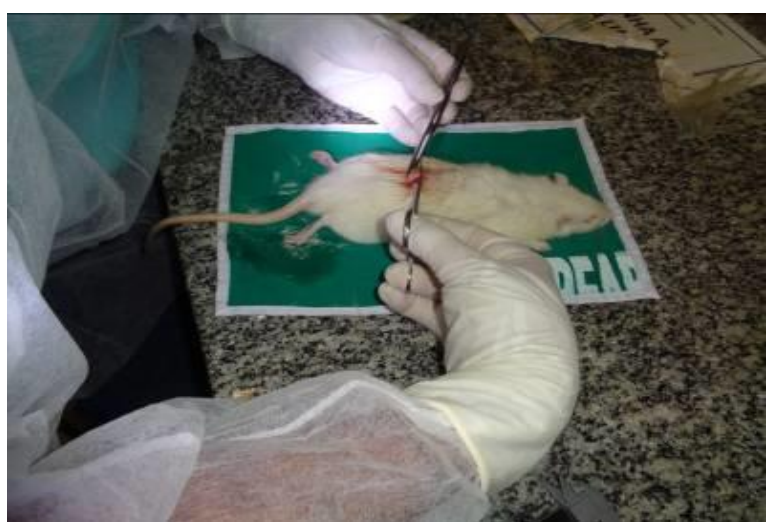

Figura 1. Fotografia demonstrando o animal em decúbito ventral sob cama cirúrgica, realizou-se na pele uma ferida a partir da incisão de um quadrante de um centímetro. com $75 \mathrm{mg} / \mathrm{kg}$ de cloridrato de cetamina associada a $10 \mathrm{mg} / \mathrm{kg}$ cloridrato de xilazina a $2 \%$ por via intramuscular com respiração espontânea. Após serem posicionados em decúbito ventral sob cama cirúrgica adaptada com seus membros em extensão, no dorso do animal se procedeu a tricotomia e antissepsia da área com digluconato de clorexidina a $2 \%$ (Figura 1). Na sequência realizouse na pele uma ferida a partir da incisão de um quadrante de um centímetro com o uso de bisturi acoplado à lâmina $n^{\circ} 24$ (Figura 2).

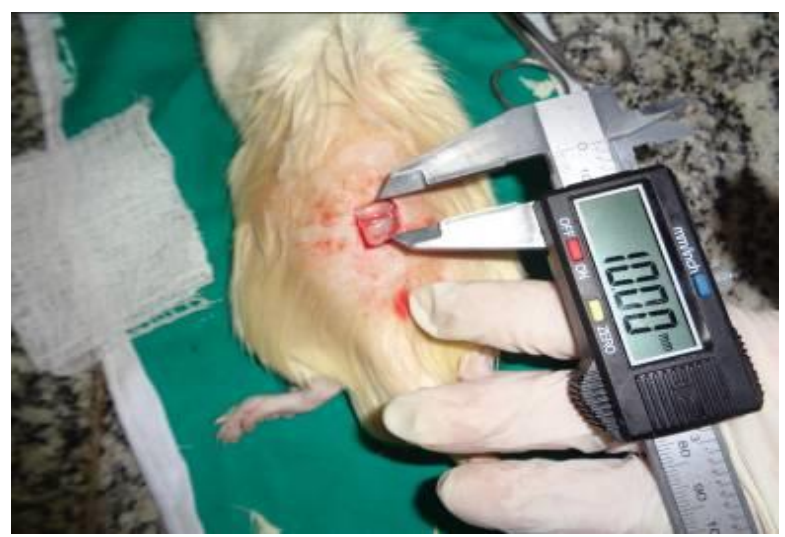

Figura 2. Fotografia evidenciando o uso do paquímetro digital para medicação da ferida cutânea realizada cirurgicamente. 
Os animais permaneceram em observação no período de recuperação anestésica, para retorno da movimentação ativa e/ou estimulada. Foram examinados clinicamente por 30 dias para acompanhar a retração da ferida cutânea por mensuração no sentido crânio-caudal com auxílio de um paquímetro digital da marca Stainless Hardened.

$\mathrm{O}$ pesquisador registrou as imagens do processo de cicatrização através de fotos com máquina fotográfica digital marca Sony Cyber-shot dsc-w830 Lcd $2.7 \quad 20 \quad 1 \mathrm{mp}$ Top, compacta, resolução de 20,1 MP, zoom óptico de $8 \mathrm{x}, \quad$ sensor $\quad$ compacto CCD, flash embutido. Mantida a uma distância para evitar imagens desfocadas, deslocando suavemente a câmera até que o sistema de lentes identificou o foco da lesão fotográfica, atendendo às recomendações para captação de imagens dermatológicas descritas em Vallarelli (2011).

O aparelho de ultrassom terapêutico pulsátil (Sonic Compact 1-3 MHz) foi calibrado em $3 \mathrm{MHz}$ para cicatrização inicial de lesão superficial conforme indicado por Johns (2002) e Kitchen (2003), Mode 100-20MHz (frequência) oscilando, potência $0,3 \mathrm{~W} / \mathrm{cm} 2$ (Olsson et al., 2008). Aplicado sob a ferida por 1 minuto em movimentos circulares com os animais contidos fisicamente. Para facilitar a utilização do transdutor, criou-se um dispositivo a partir de uma lupa (Magnifying Glass $75 \mathrm{~mm}$ ), onde a lente foi trocada por uma película de filme plástico formando uma membrana de uso individual para evitar contaminações e facilitar a aplicação do gel de contato (Figura 3).

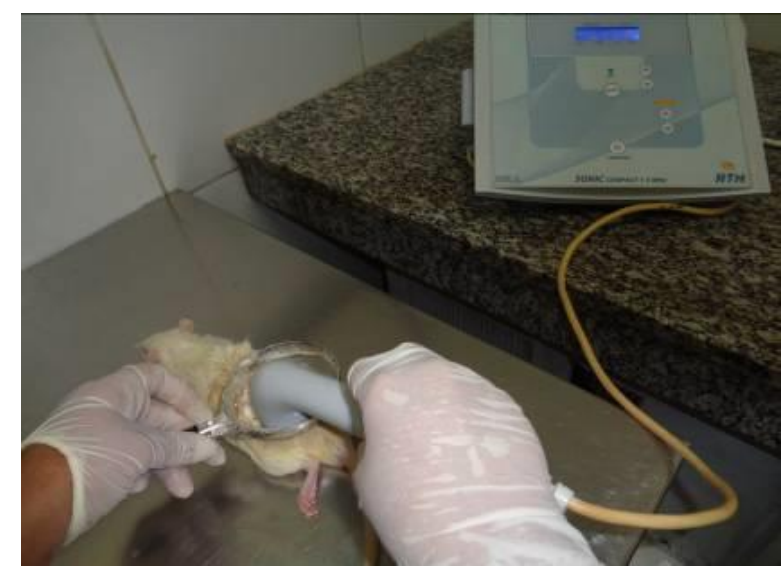

Figura 3. Fotografia evidenciando a aplicação do ultrassom. Para facilitar a aplicação, criouse um equipamento/dispositivo a partir de uma lupa, onde a lente foi substituída por uma película de filme plástico.
O aparelho de ultrassom terapêutico foi calibrado e submetido à dosimetria antes do início do tratamento para evitar erros de dosagem, e o cabeçote adaptado para pequenas áreas, segundo as orientações de Young (1990).

Os resultados de cada grupo foram submetidos à análise estatística onde primeiramente se verificou se existiu diferença significativa entre os tratamentos por meio do teste F. Posteriormente, avaliou-se a magnitude destas diferenças utilizando um teste de comparações múltiplas. O teste de Tukey foi selecionado por permitir testar qualquer contraste, sempre entre duas médias de tratamentos.

\section{Resultados}

Os animais não apresentaram complicações durante $\mathrm{o}$ ato operatório ou dele decorrentes durante todo o período de 30 dias de observação experimental. No tocante aos efeitos colaterais, os animais tratados com o óleo de semente de girassol não apresentaram nenhum tipo de reação tópica adversa ao produto.

Por se tratar de um experimento inteiramente casualizado, primeiramente se verificou se houve diferença significativa entre os tratamentos, por meio do teste F. Como o F calculado (53.3396) foi maior que o $\mathrm{F}$ tabelado, o teste já o insere como significativo ao nível de $1 \%$. Com isso, existiu diferença significativa em pelo menos um par de tratamentos, sendo assim analisou-se em qual tratamento através do Teste Tukey-Kramer. Após constatar que houve diferença significativa entre os tratamentos, por meio do teste $\mathrm{F}$, avaliou-se a magnitude destas diferenças utilizando um teste de comparações múltiplas. O teste de Tukey foi selecionado por permitir testar qualquer contraste, sempre entre duas médias de tratamentos.

Considerando o efeito de tempo para cicatrização total das feridas nos animais, verificou-se forte significância $(p<0,001)$ no grupo III (ultrassom e óleo de semente de girassol), evidenciados os resultados por meio de gráficos de colunas com a análise dos dados de cada grupo por tempo de cicatrização (Figura 4).

De acordo com o teste $\mathrm{F}$, foram encontradas evidências de diferenças significativas, ao nível de $1 \%$ de probabilidade, entre os tratamentos, com relação ao tempo médio de cicatrização. Apenas não tendo sido encontrada diferença significativa nos tempos entre $o$ tratamento com óleo de semente de girassol (Grupo 
II) e o tratamento apenas com o ultrassom (Grupo IV), mas a grande significância da diferença nos tempos foi encontrada quando utilizado o ultrassom em conjunto com o óleo de semente de girassol (Grupo III) em relação aos demais grupos (Tabela 3).

Para todos os grupos de tratamento com uma média de oito dias já havia $50 \%$ de cicatrização das feridas, enquanto para o grupo I (controle) apenas com uma média de $11 \pm 2,60$ dias, mas o grupo III com uma média de apenas 5,17 $\pm 0,76$ dias.

Ao fim do experimento os animais foram encaminhados para rotina do biotério, sem realização de eutanásia, preservando pela vida do animal.

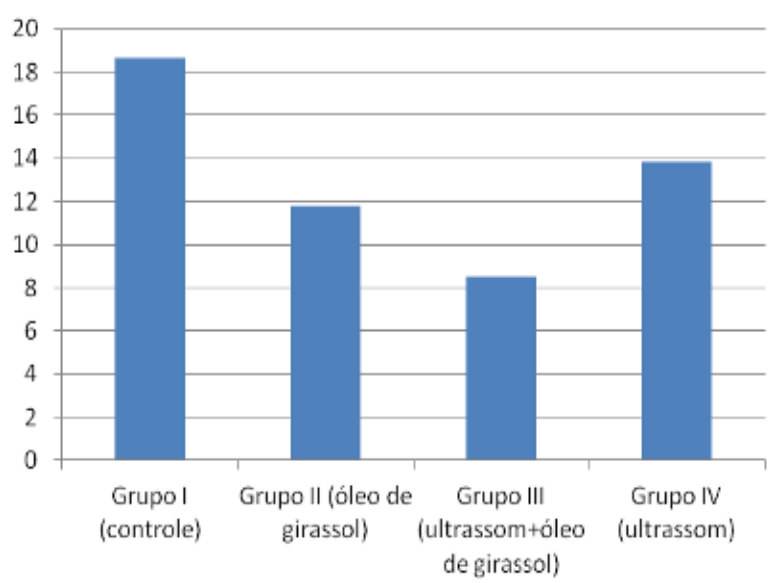

Figura 4. Gráfico evidenciando a média de tempo de cicatrização total entre os grupos.

Tabela 3. Análise individual do tempo (dias) de cicatrização total das feridas.

\begin{tabular}{lcccc}
\hline Animal & Grupo I & Grupo II & Grupo III & Grupo IV \\
\hline Animal I & 22 & 12 & 10 & 14 \\
Animal II & 17 & & 9 & 12 \\
Animal III & 17 & 12 & 9 & 14 \\
Animal IV & 18 & 11 & 8 & 12 \\
Animal V & 19 & 13 & 7 & 15 \\
Animal VI & 19 & 11 & 8 & 16 \\
\hline Média de tempo/ cicatrização total & $18,67^{\mathrm{a}}$ & $11,80^{\mathrm{b}}$ & $8,50^{\mathrm{c}}$ & $13,83^{\mathrm{b}}$ \\
\hline
\end{tabular}

Anova: Efeito do tempo significativo $\mathrm{p}<0,01$.

Teste Tukey-Kramer: médias com letras diferentes indicam que houve uma diferença ao nível de 0,05 de significância. Onde as letras a, b e c colocadas pela ferramenta traduz que as médias com letras diferentes houve uma diferença ao nível de 0,05 de significância.

\section{Discussão}

Foram administrados cetamina e xilazina como anestésicos de eleição, também utilizados por Modolin e Kamakura (2009) e Ballaben et al. (2013) em suas pesquisas com ratos e ratas, respectivamente.

A realização da ferida com apenas $1 \mathrm{~cm}^{2}$ permitiu a visualização macroscópica de todo processo cicatricial e minimizou os seus efeitos quanto ao bem-estar, por ser uma área de pequena extensão. Por outro lado, com $4 \mathrm{~cm}^{2}$ Bem et al. (2010) analisaram histologicamente o tecido epitelial sadio de ratos Wistar (in vivo) irradiados com diferentes intensidades do ultrassom.

Diferente de Bem et al. (2010) que realizaram um período de adaptação por dois dias ao uso do ultrassom nos seus animais, não foi preciso este procedimento no presente trabalho. Além do que, a partir do $5^{\circ}$ dia de aplicação do ultrassom os animais apresentaram relaxamento e com o auxílio do dispositivo confeccionado para facilitar a utilização do transdutor não foi necessária uma contenção traumática dos animais.

A frequência do transdutor utilizada foi de 3 $\mathrm{MHz}$, sendo esta mais superficial e considerada uma frequência terapêutica, com profundidade de $1 \mathrm{a} 2 \mathrm{~cm}$ conforme descreveram Johns (2002) e Kitchen (2003). Assim como Mendonça et al. (2006), a escolha desta intensidade dá-se ao seu efeito benéfico sem gerar aquecimento ou calor sobre a ferida. Ainda, de acordo com Guirro (1995) o ultrassom terapêutico no modo pulsado não gera calor o suficiente para ocasionar alterações biológicas nos tecidos e que a onda ultrassônica do modo pulsado provoca efeito mecânico e não térmico. E segundo Freitas et al. (2011) sendo os efeitos não térmico benéficos no processo de cicatrização. A frequência de $3 \mathrm{MHz}$ utilizada corroborou os estudos de Bem et al. (2010) e Guimarães et al. (2011).

A potência utilizada $0,3 \mathrm{~W} / \mathrm{cm}^{2}$, segundo Blume et al. (2005) e Olsson et al. (2008), que relataram existir equipamentos reprojetados para apresentar limites de intensidade mais compatíveis com a prática clínica, que variam de 0,1 a $2,0 \mathrm{~W} / \mathrm{cm}^{2}$, uma vez que raramente se utilizam doses superiores a $2,0 \mathrm{~W} / \mathrm{cm}^{2}$.

Com um minuto de aplicação do ultrassom sob a ferida, como sugerido por Guimarães et al. (2011), obteve-se os resultados da pesquisa, no 
entanto, diferentes tempos de dois e cinco minutos de aplicação foram realizados por Bem et al. (2010), Freitas e Prado (2015), respectivamente.

Durante a avaliação clínica se observou nas primeiras 48 horas retração da ferida, hiperemia e neovascularização. $\mathrm{O}$ ultrassom pode promover angiogênese, regeneração tissular, reparação de tecidos moles, aumento na circulação sanguínea e alívio a dor, como observado por Saini et al. (2002) e Matheus et al. (2008).

\section{Conclusão}

A aplicação do ultrassom terapêutico favoreceu a permeabilidade do óleo de semente de girassol no tecido lesado e consequentemente melhorou o processo de reparação com diminuição do tempo de cicatrização de feridas cutâneas induzidas em ratos. O presente estudo abordou o aspecto macroscópico da regeneração tegumentar, seus resultados vêm complementar outros trabalhos, com isso tem-se a perspectiva que este experimento sirva de subsídio para pesquisas futuras com outras espécies visando um melhor tratamento de feridas cutâneas.

\section{Conflito de Interesse}

Os autores declaram não existir conflito de interesse.

\section{Comitê de Ética}

Este estudo foi realizado no biotério do Departamento de Morfologia e Fisiologia Animal da Universidade Federal Rural de Pernambuco, sob licença do Comitê de Ética $\mathrm{n}^{\circ}$ 016/2016 (CEUA/UFRPE).

\section{Referências}

Ballaben, A.S.; Crisci, A.R.; Jorge, M.H.S. Efeito da pomada de barbatimão (Stryphnodendrom barbatiman Martius) associado ao ultrassom de baixa intensidade sobre a cicatrização por segunda intenção de lesões cutâneas totais em ratos. Revista ReBraM, 16(1): 159-172, 2013.

Bem, D.M.D.; Maciel, C.D.; Zuanon, J.A.; Neto, C.B.; Parizotto, N.A. Análise histológica em tecido epitelial sadio de ratos Wistar (in vivo) irradiados com diferentes intensidades do ultrassom. Revista Brasileira de Fisioterapia, 14(2): 114-120, 2010.

Benson, H.A.E.; McElnay, J.C. Transmissão de energia ultra-sônica através de produtos farmacêuticos tópicos. Physiotherapy, 74(11): 587-588, 1988.
Blume, K.; Matsuo, E.; Lopes, M.S.; Lopes, L.G. Dosimetria proposta para o tratamento por ultrassom: uma revisão de literatura. Fisioterapia em Movimento, 18(3): 55-64, 2005.

Freitas, I.S.; Prado, L.G. Utilização do ultrassom terapêutico e do óleo de semente de girassol na cicatrização de feridas cutâneas em equinos. Revista Científica da FEPI, 8(2): 1-3, 2015.

Freitas, T.P.; Freitas, L.S.; Streck, E.L. Ultrassom terapêutico no mecanismo de cicatrização: uma revisão. Arquivos Catarinenses de Medicina, 40(1): 89-93, 2011.

Guimarães, G.N.; Pires-de-Campos, M.S.M.; Leonardi, G.R.; Dib-Giusti, H.H.K.; Polacow, M.L.O. Efeito do ultrassom e do dexapantenol na organização das fibras colágenas em lesão tegumentar. Brazilian Journal of Physical Therapy, 15(3): 227-232, 2011.

Guirro, E.C.O.; Guirro, E.; Ferreira, A.L. Efeitos da estimulação ultra-sônica pulsada de baixa intensidade no processo cicatricial: estudo experimental em ratos. Revista Ciência e Tecnologia, 8: 37-47, 1995.

Johns, L.D. Nonthermal effects of therapeutic ultrasound: the frequency resonance hyposthesis. Journal of Athletic Training, 37(3): 293-299, 2002.

Kitchen, S. Eletroterapia: prática baseada em evidências. $11^{\mathrm{a}}$ ed. São Paulo: Manole, 2003. $711 \mathrm{p}$.

Kitchen, S.S.; Partridge, C.J. A review of therapeutic ultrasound: II. The efficacy of ultrasound. Physiotherapy, 76: 593-595, 1990.

Korelo, R.I.G.; Fernandes, L.C. Ultrassom terapêutico para cicatrização de feridas: revisão sistemática. ConScientiae Saúde, 15(3): 518529, 2016.

Lania, B.G. O uso dos óleos de café e girassol em feridas cutâneas: efeitos locais e sistêmicos. Tese (doutorado). Universidade Estadual de Campinas, Faculdade de Ciências Médicas, Campinas, SP. Unicamp, 2018. 125p.

Leite, A.P.; de Oliveira, B.G.R.P.; Soares, M.F.; Barrocas, D.L.R. Uso e efetividade da papaína no processo de cicatrização de feridas: uma revisão sistemática. Revista Gaúcha de Enfermagem, 33(3): 198-207, 2012.

Marques, S.R.; Peixoto, C.A.; Messias, J.B.; de Albuquerque, A.R.; da Silva, J.V.A. The effects of topical application of sunflower-seed oil on open wound healing in lambs. Acta Cirurgia Brasileira, 19(3): 196-209, 2004. 
Marks, R.; Ghanagaraja, S.; Ghassemi, M. Ultrasound for osteo-arthritis of the knee. Physioterapy, 86(9): 454-463, 2000.

Matheus, J.P.C.; Oliveira, F.B.; Gomide, L.B.; Milani, J.G.P.O.; Volpon, J.B.; Shimano, A.C. Efeitos do ultrassom terapêutico nas propriedades mecânicas do músculo esquelético após contusão. Revista Brasileira de Fisioterapia, 12(3): 241-247, 2008.

Mendonça, A.C.; Ferreira, A.S.; Barbieri, C.H.; Thomazine, J.A.; Mazzer, N. Efeitos do ultrasom pulsado de baixa intensidade sobre a cicatrização por segunda intenção de lesões cutâneas totais em ratos. Acta Ortopédica Brasileira, 14(3): 152, 2006.

Modolin, M.; Kamakura, L. Aspectos gerais da cicatrização das feridas. In: Sucena, R.C.; Oliveira, H.C.S.; Vieira, D.; Ferreira, C.M.R. Avaliação do potencial cicatricial do gel de confrei (Symphytum officinale L.) associado à técnica do ultrassom terapêutico em ratos com feridas cutâneas. Revista Núcleo Interdisciplinar de Pesquisa e Extensão do UNIPAM, 15(6): 31-44, 2009.

Olsson, D.C.; Martins, V.M.V.; Pippi, N.L.; Mazzanti, A.; Tognoli, G.K. Ultra-som terapêutico na cicatrização tecidual. Ciência Rural, 38(4): 1199-1207, 2008.
Roger, M.; Fullard, N.; Costello, L.; Bradbury, S.; Markiewicz, E.; O'Reilly, S.; Darling, N.; Ritchie, P.; Määttä, A.; Karakesisoglou, I.; Nelson, G.; von Zglinicki, T.; Dicolandrea, T.; Isfort, R.; Bascom, C.; Przyborski, Stefan. Bioengineering the microanatomy of human skin. Journal of Anatomy, 234(4): 438-455, 2019.

Saini, N.S.; Roy, K.S.; Bansal, P.S.; Singh, B.; Simran, P.S. A preliminary study on the effect of ultrasound therapy on the healing of surgically severed achilles tendons in five dogs. Journal of Veterinary Medicine Series A, 49(6): 321-328, 2002.

Sousa, R.D.S. Estudo de substâncias químicas em óleos de coco, copaíba, calêndula e girassol utilizados no tratamento de feridas: uma abordagem teórica. 2018. Monografia. Disponível em: <http://hdl.handle.net/123456789/2357>. Acesso em: 25 abr. 2020.

Vallarelli, A.F. Critérios para submissão de fotografias. Anais da Sociedade Brasileira de Dermatologia, 86: 212-214, 2011.

Young, S.R.; Dyson, M. The effect of therapeutic ultrasound on angiogenesis. Ultrasound in Medicine \& Biology, 16(3): 261-269, 1990. 